\title{
Fast 3D Pattern Synthesis with Polarization and Dynamic Range Ratio Control for Conformal Antenna Arrays
}

\author{
Massimiliano Comisso and Roberto Vescovo \\ Department of Engineering and Architecture, University of Trieste, Via A. Valerio 10, 34127 Trieste, Italy \\ Correspondence should be addressed to Massimiliano Comisso; mcomisso@units.it
}

Received 5 March 2014; Revised 24 June 2014; Accepted 3 July 2014; Published 17 July 2014

Academic Editor: Sembiam R. Rengarajan

Copyright ( 2014 M. Comisso and R. Vescovo. This is an open access article distributed under the Creative Commons Attribution License, which permits unrestricted use, distribution, and reproduction in any medium, provided the original work is properly cited.

\begin{abstract}
This paper proposes an iterative algorithm for the 3D synthesis of the electric far-field pattern of a conformal antenna array in the presence of requirements on both the polarization and the dynamic range ratio (DRR) of the excitations. Thanks to the use of selectable weights, the algorithm allows a versatile control of the DRR and of the polarization in a given angular region and requires a low CPU time to provide the array excitations. Furthermore, a modified version of the algorithm is developed to enable the optimization of the polarization state by phase-only control. Numerical results are presented to verify the usefulness of the proposed approach for the joint pattern and polarization synthesis of conformal arrays with reduced or even unitary DRR.
\end{abstract}

\section{Introduction}

The polarization properties of an antenna array can be exploited to acquire considerable benefits, such as mitigating fading effects, improving the performance of a radar system, and compensating the transmitter/receiver polarization mismatch. To obtain a desired polarization, an approach widely adopted by the antenna developers relies on the design of the physical antenna system, thus operating on the array geometry [1], on the technology of the single array element $[2,3]$, and on the position of the feeds [4] or, more in general, using combinations of these techniques [5-8]. When the physical antenna system is given, including the elements, the geometry, and the feeding network, the designer can act on the array excitations by properly selecting their amplitudes and phases in order to satisfy the polarization requirements. Accordingly, different solutions have been presented in the literature for minimizing the cross-polar pattern, when a linear polarization is desired [9-11], or for enabling the generation of more specific circular or even elliptical polarizations $[12,13]$. Furthermore, in addition to the polarization requirements, the array must be often able to generate a desired pattern in the presence of constraints on the dynamic range ratio (DRR) of the excitations. For the case in which no constraints on the DRR are imposed, the authors of [9] describe an algorithm based on the alternating projection method and the use of an Euler rotation matrix to provide low cross-polarization patterns for conformal arrays. In [10], the array synthesis in the presence of requirements on the cross-polarization level and the side lobe level (SLL) is performed by combining particle swarm optimization and alternate projection methods. A technique for simultaneously synthesizing the copolar and the cross-polar array amplitude patterns in the presence of DRR requirements is proposed in [11]. A least-square optimization approach is adopted in [12] for synthesizing the pattern of a conformal array imposing a given polarization and a specified value for each excitation amplitude. The joint synthesis of an upper bounded pattern and a desired polarization in a given angular region for a conformal array in absence of DRR constraints is addressed in [13] by adopting a convex formulation of the synthesis problem, which is solved by a second-order cone program.

This overview reveals that the problem of controlling the DRR together with the polarization of the electric far-field vector of the array is still open. To deal with such problem, this study proposes a versatile method for the 3D pattern synthesis of a conformal array, when DRR reduction and polarization control in a given angular region are simultaneously required. The method extends the approach used in [11], whose application is limited to linear polarizations, to enable 
the generation of any desired polarization while maintaining a low synthesis CPU time. Moreover, with respect to [11], a modified version of the method is developed to solve the problem of joint pattern and polarization synthesis by phaseonly control.

The paper is organized as follows. Section 2 introduces the problem. Section 3 presents the algorithm and its modification for phase-only control. Section 4 discusses the numerical results. Section 5 summarizes the main conclusions.

\section{Problem Formulation}

In the $3 \mathrm{D}$ space, referred to as a spherical coordinate system $O(r, \theta, \phi)$, a conformal array of $N$ elements and the column vector of its complex excitations $\mathbf{a}=\left[a_{1}, \ldots, a_{N}\right]^{T}$ is considered, with $(\cdot)^{T}$ denoting the transpose operator. Such excitations generate the electric far-field patterns $E_{\theta}(\mathbf{a} ; \widehat{\mathbf{r}})$ and $E_{\phi}(\mathbf{a} ; \widehat{\mathbf{r}})$, where $\widehat{\mathbf{r}}$ is the unit vector of the direction of observation, while $E_{\theta}(\mathbf{a} ; \widehat{\mathbf{r}})$ and $E_{\phi}(\mathbf{a} ; \widehat{\mathbf{r}})$ are the components, along the standard unit vectors $\widehat{\boldsymbol{\theta}}$ and $\widehat{\phi}$, respectively, of the electric far-field vector $\mathbf{E}(\mathbf{a} ; \widehat{\mathbf{r}})$; that is, $\mathbf{E}(\mathbf{a} ; \widehat{\mathbf{r}})=E_{\theta}(\mathbf{a} ; \widehat{\mathbf{r}}) \widehat{\boldsymbol{\theta}}+$ $E_{\phi}(\mathbf{a} ; \widehat{\mathbf{r}}) \widehat{\phi}$. Thus, we can write

$$
\begin{aligned}
E_{\theta}(\mathbf{a} ; \widehat{\mathbf{r}}) & =\sum_{n=1}^{N} a_{n} \widetilde{\mathscr{E}}_{n}(\widehat{\mathbf{r}}) e^{j(2 \pi / \lambda) \overline{\mathbf{r}}_{n} \cdot \widehat{\mathbf{r}}} \\
& =\sum_{n=1}^{N} a_{n} \widetilde{f}_{n}(\widehat{\mathbf{r}})=\mathbf{a}^{T} \widetilde{\mathbf{f}}(\widehat{\mathbf{r}}), \\
E_{\phi}(\mathbf{a} ; \widehat{\mathbf{r}}) & =\sum_{n=1}^{N} a_{n} \widehat{\mathscr{E}}_{n}(\widehat{\mathbf{r}}) e^{j(2 \pi / \lambda) \overline{\mathbf{r}}_{n} \cdot \widehat{\mathbf{r}}} \\
& =\sum_{n=1}^{N} a_{n} \widehat{f}_{n}(\widehat{\mathbf{r}})=\mathbf{a}^{T} \widehat{\mathbf{f}}(\widehat{\mathbf{r}}),
\end{aligned}
$$

where $\widetilde{\mathscr{E}}_{n}(\widehat{\mathbf{r}})$ and $\widehat{\mathscr{E}}_{n}(\widehat{\mathbf{r}})$ are the components along $\widehat{\boldsymbol{\theta}}$ and $\widehat{\phi}$, respectively, of the electric far-field vector of the $n$th array element, $\overline{\mathbf{r}}_{n}$ is the position of the $n$th element, $\lambda$ is the wavelength, $\widetilde{\mathbf{f}}(\widehat{\mathbf{r}})=\left[\tilde{f}_{1}(\widehat{\mathbf{r}}), \ldots, \widetilde{f}_{N}(\widehat{\mathbf{r}})\right]^{T}$, and $\widehat{\mathbf{f}}(\widehat{\mathbf{r}})=$ $\left[\widehat{f}_{1}(\widehat{\mathbf{r}}), \ldots, \widehat{f}_{N}(\widehat{\mathbf{r}})\right]^{T}$. Consider now a positive function $E(\widehat{\mathbf{r}})$ normalized to unity, and a pair of coefficients $(\delta, \xi)$, with $\delta \geq 0$ and $-\pi \leq \xi \leq \pi$, which specifies a desired polarization for $\mathbf{E}(\mathbf{a} ; \widehat{\mathbf{r}})$. The objective is to find an excitation vector a such that

$$
\left|E_{\theta}(\mathbf{a} ; \widehat{\mathbf{r}})\right| \text { approximates } E(\widehat{\mathbf{r}}) ;
$$

$\mathbf{E}(\mathbf{a} ; \widehat{\mathbf{r}})$ is polarized according to $(\delta, \xi)$; that is,

$E_{\theta}(\mathbf{a} ; \widehat{\mathbf{r}})=E_{\phi}(\mathbf{a} ; \widehat{\mathbf{r}}) \delta e^{j \xi}$ in a given angular region,

$$
\operatorname{DRR}(\mathbf{a})=\frac{\max _{n}\left\{\left|a_{n}\right|\right\}}{\min _{n}\left\{\left|a_{n}\right|\right\}} \text { is reduced. }
$$

As remarked in [11, 13-15], one can observe that the expressions in (1a) and (1b) include the mutual coupling effects between the array elements, provided that the singleelement patterns $\widetilde{\mathscr{E}}_{n}(\widehat{\mathbf{r}})$ and $\widehat{\mathscr{E}}_{n}(\widehat{\mathbf{r}})$ are numerically calculated by using a suitable software. Precisely, following [15], the patterns $\widetilde{\mathscr{E}}_{n}(\widehat{\mathbf{r}})$ and $\widehat{\mathscr{E}}_{n}(\widehat{\mathbf{r}})$ can be evaluated by considering the $n$th element as fed by unity voltage and zero phase, and the other $N-1$ array elements without feeds. Thus, $\widetilde{\mathscr{E}}_{n}(\widehat{\mathbf{r}})$ and $\widehat{\mathscr{E}}_{n}(\widehat{\mathbf{r}})$ do not identify the isolated element patterns but identify the equivalent element patterns accounting for the coupling with the remaining array elements. The possibility of using this approach is enabled by the linear relationships in ((1a) and (1b)) between the electric far-field patterns of the single-element and those generated by the array [11, 13-15].

\section{Synthesis Algorithm}

To solve the above formulated problem, we introduce the following cost function:

$$
\begin{aligned}
\mathscr{F}(\mathbf{a})= & \int_{\Theta}|| E_{\theta}(\mathbf{a} ; \widehat{\mathbf{r}})\left|-\frac{E(\widehat{\mathbf{r}})}{w(\widehat{\mathbf{r}})}\right|^{2} w(\widehat{\mathbf{r}}) \mathrm{d} \theta \mathrm{d} \phi \\
& +\int_{\Theta_{\mathrm{ML}}}\left|E_{\theta}(\mathbf{a} ; \widehat{\mathbf{r}})-E_{\phi}(\mathbf{a} ; \widehat{\mathbf{r}}) \delta e^{j \xi}\right|^{2} v(\widehat{\mathbf{r}}) \mathrm{d} \theta \mathrm{d} \phi \\
& +b \sum_{m, n=1}^{N} \frac{\left(\left|a_{n}\right|-\left|a_{m}\right|\right)^{2}}{N},
\end{aligned}
$$

where $\Theta$ and $\Theta_{\text {ML }}$ denote the solid angular regions that identify the entire domain of interest and the main lobe, respectively, $w(\widehat{\mathbf{r}})$ and $v(\widehat{\mathbf{r}})$ are positive weight functions, and $b$ is a nonnegative weight. Minimizing the first term in (3) allows the amplitude of $E_{\theta}(\mathbf{a} ; \widehat{\mathbf{r}})$ to approximate $E(\widehat{\mathbf{r}})$. Minimizing the second term allows one to obtain a pattern $E_{\phi}(\mathbf{a} ; \widehat{\mathbf{r}})$ approximately scaled by $1 / \delta$ and with a phase shift $-\xi$ with respect to $E_{\theta}(\mathbf{a} ; \widehat{\mathbf{r}})$, thus providing the desired polarization. Minimizing the third term reduces the DRR of the excitations.

Now, starting from the desired positive function $E(\widehat{\mathbf{r}})$, let us consider the complex function $E(\widehat{\mathbf{r}}) e^{j \Phi(\widehat{\mathbf{r}})}$, where $\Phi(\widehat{\mathbf{r}})$ is an arbitrary real function. Then, minimizing the first integral in (3) is equivalent to minimizing the new integral $\int_{\Theta}\left|E_{\theta}(\mathbf{a} ; \widehat{\mathbf{r}})-E(\widehat{\mathbf{r}}) e^{j \Phi(\widehat{\mathbf{r}})} w^{-1}(\widehat{\mathbf{r}})\right|^{2} w(\widehat{\mathbf{r}}) \mathrm{d} \theta \mathrm{d} \phi$. Note that the function $\Phi(\widehat{\mathbf{r}})$ minimizing the latter integral coincides with the phase function of $E_{\theta}(\mathbf{a} ; \widehat{\mathbf{r}})$. Thus, our problem can be solved by minimizing the functional

$$
\begin{aligned}
d^{2}[\mathbf{a}, \Phi(\widehat{\mathbf{r}})]= & \int_{\Theta}\left|E_{\theta}(\mathbf{a} ; \widehat{\mathbf{r}})-\frac{E(\widehat{\mathbf{r}}) e^{j \Phi(\widehat{\mathbf{r}})}}{w(\widehat{\mathbf{r}})}\right|^{2} w(\widehat{\mathbf{r}}) \mathrm{d} \theta \mathrm{d} \phi \\
& +\int_{\Theta_{\mathrm{ML}}}\left|E_{\theta}(\mathbf{a} ; \widehat{\mathbf{r}})-E_{\phi}(\mathbf{a} ; \widehat{\mathbf{r}}) \delta e^{j \xi}\right|^{2} v(\widehat{\mathbf{r}}) \mathrm{d} \theta \mathrm{d} \phi \\
& +b \sum_{m, n=1}^{N} \frac{\left(\left|a_{n}\right|-\left|a_{m}\right|\right)^{2}}{N} .
\end{aligned}
$$

Thus, the power synthesis problem at hand is reformulated as a field synthesis problem consisting in the minimization of 
(4). The unknowns a and $\Phi(\widehat{\mathbf{r}})$ minimizing $d^{2}$ can be found recalling the procedure used in [11]. As a first step, (4) can be rewritten as

$$
\begin{aligned}
d^{2}[\mathbf{a}, \Phi(\widehat{\mathbf{r}})]= & \int_{\Theta}\left|E_{\theta}(\mathbf{a} ; \widehat{\mathbf{r}})\right|^{2} w(\widehat{\mathbf{r}}) \mathrm{d} \theta \mathrm{d} \phi \\
& -\int_{\Theta} E(\widehat{\mathbf{r}})\left[E_{\theta}(\mathbf{a} ; \widehat{\mathbf{r}}) e^{-j \Phi(\widehat{\mathbf{r}})}\right. \\
& \left.\quad+E_{\theta}^{*}(\mathbf{a} ; \widehat{\mathbf{r}}) e^{j \Phi(\widehat{\mathbf{r}})}\right] \mathrm{d} \theta \mathrm{d} \phi \\
& +\int_{\Theta_{\mathrm{ML}}}\left|E_{\theta}(\mathbf{a} ; \widehat{\mathbf{r}})-E_{\phi}(\mathbf{a} ; \widehat{\mathbf{r}}) \delta e^{j \xi}\right|^{2} v(\widehat{\mathbf{r}}) \mathrm{d} \theta \mathrm{d} \phi \\
& +\frac{2 b}{N}\left(N \sum_{n=1}^{N}\left|a_{n}\right|^{2}-\sum_{m, n=1}^{N}\left|a_{n} a_{m}\right|\right)+\Upsilon^{2},
\end{aligned}
$$

where $(\cdot)^{*}$ is the complex conjugate and

$$
\Upsilon^{2}=\int_{\Theta} \frac{E^{2}(\widehat{\mathbf{r}})}{w(\widehat{\mathbf{r}})} \mathrm{d} \theta \mathrm{d} \phi .
$$

Assume now the following notations: $(\cdot)^{H}$ is the Hermitian operator, $\mathfrak{R}(\cdot)$ is the real part, $\|\cdot\|_{F}$ is the Frobenius norm, and $\|\cdot\|_{1}$ is the sum of the moduli of the elements of a matrix or vector, thus representing the entrywise absolute norm for a matrix and the Manhattan norm for a vector. Substituting (1a) and (1b) into (5) and manipulating, one obtains

$$
\begin{aligned}
d^{2}[\mathbf{a}, \Phi(\widehat{\mathbf{r}})]= & \mathbf{a}^{H} \mathbf{A a}-2 \mathfrak{R}\left(\mathbf{g}^{H} \mathbf{a}\right) \\
& -\frac{2 b}{N}\left\|\mathbf{a} \mathbf{a}^{H}\right\|_{1}+2 b\|\mathbf{a}\|_{F}^{2}+\Upsilon^{2},
\end{aligned}
$$

where $\mathbf{A}=\left[A_{m n}\right], \mathbf{g}=\left[g_{1}, \ldots, g_{N}\right]^{T}$, and

$$
\begin{aligned}
A_{m n}=\int_{\Theta} \tilde{f}_{n}^{*}(\widehat{\mathbf{r}}) \tilde{f}_{m}(\widehat{\mathbf{r}}) w(\widehat{\mathbf{r}}) \mathrm{d} \theta \mathrm{d} \phi \\
+\int_{\Theta_{\mathrm{ML}}}\left\{-\delta\left[\widehat{f}_{n}^{*}(\widehat{\mathbf{r}}) \tilde{f}_{m}(\widehat{\mathbf{r}}) e^{-j \xi}+\widetilde{f}_{n}^{*}(\widehat{\mathbf{r}}) \widehat{f}_{m}(\widehat{\mathbf{r}}) e^{j \xi}\right]\right. \\
\left.\quad+\widetilde{f}_{n}^{*}(\widehat{\mathbf{r}}) \tilde{f}_{m}(\widehat{\mathbf{r}})+\delta^{2} \widehat{f}_{n}^{*}(\widehat{\mathbf{r}}) \widehat{f}_{m}(\widehat{\mathbf{r}})\right\} v(\widehat{\mathbf{r}}) \mathrm{d} \theta \mathrm{d} \phi
\end{aligned}
$$

$$
g_{n}=\int_{\Theta} \widetilde{f}_{n}^{*}(\widehat{\mathbf{r}}) E(\widehat{\mathbf{r}}) e^{j \Phi(\widehat{\mathbf{r}})} \mathrm{d} \theta \mathrm{d} \phi
$$

which can be minimized iteratively by subdividing each iteration in two parts. In the first part, given $\Phi(\widehat{\mathbf{r}}), d^{2}$ is minimized with respect to a by modifying the excitations one at a time. In particular, observing that $A_{m n}=A_{n m}^{*}$, (7) can be manipulated to put into evidence its dependence on the generic $p$ th component $a_{p}$. Thus, defining $\zeta_{p}=\left|a_{p}\right|$ and $\psi_{p}=\arg \left(a_{p}\right)$, one obtains

$$
\begin{aligned}
d^{2}[\mathbf{a}, \Phi(\widehat{\mathbf{r}})]= & 2\left\{\left|T_{p}\right| \cos \left[\psi_{p}-\arg \left(T_{p}\right)\right]-\frac{2 b}{N}\left\|\mathbf{a}_{p}\right\|_{1}\right\} \zeta_{p} \\
& +\left[A_{p p}+2 b\left(1-\frac{1}{N}\right)\right] \zeta_{p}^{2}+\chi_{p},
\end{aligned}
$$

where $T_{p}=\mathbf{A}_{p} \mathbf{a}_{p}-g_{p}$, with $\mathbf{A}_{p}$ denoting the $p$ th row of the matrix $\mathbf{A}$ and $\mathbf{a}_{p}=\left[a_{1}, \ldots, a_{p-1}, 0, a_{p+1}, \ldots, a_{N}\right]^{T}$, and where $\chi_{p}$ is a term independent of $a_{p}$. Now, (9) can be minimized with respect to the argument $\psi_{p}$ and to the amplitude $\zeta_{p}$. Precisely, imposing that the multiplicative coefficient of $\zeta_{p}$ be minimum, one obtains $\psi_{p}=\arg \left(T_{p}\right)+(2 \kappa+1) \pi$, where $\kappa$ is an arbitrary integer, while imposing that the derivative of $d^{2}$ with respect to $\zeta_{p}$ be zero, one obtains $\zeta_{p}=$ $\left(N\left|T_{p}\right|+2 b\left\|\mathbf{a}_{p}\right\|_{1}\right) /\left[N A_{p p}+2 b(N-1)\right]$. Combining these two expressions, the value of $a_{p}=\zeta_{p} e^{j \psi_{p}}$ minimizing (9) can be written in compact form as

$$
a_{p}=-\frac{2 b e^{j \arg \left(T_{p}\right)}\left\|\mathbf{a}_{p}\right\|_{1}+N T_{p}}{N A_{p p}+2 b(N-1)} .
$$

In the second part of each iteration, given $\mathbf{a}, d^{2}$ is minimized with respect to $\Phi(\widehat{\mathbf{r}})$. Precisely, by substituting (8b) into (7), one obtains

$$
d^{2}[\mathbf{a}, \Phi(\widehat{\mathbf{r}})]=\eta-2 \Re\left[\int_{\Theta} \mathbf{a}^{T} \widetilde{\mathbf{f}}(\widehat{\mathbf{r}}) E(\widehat{\mathbf{r}}) e^{-j \Phi(\widehat{\mathbf{r}})} \mathrm{d} \theta \mathrm{d} \phi\right]
$$

which, being the term $\eta$ independent of $\Phi(\widehat{\mathbf{r}})$, is minimized for

$$
\Phi(\widehat{\mathbf{r}})=\arg \left[\mathbf{a}^{T} \widetilde{\mathbf{f}}(\widehat{\mathbf{r}})\right] .
$$

Summarizing, the proposed algorithm for polarization and DRR control develops as follows. Moving from a starting point $\mathbf{a}^{0}$, the function $\Phi^{0}(\widehat{\mathbf{r}})$ is evaluated by (12). At each successive iteration $q=1,2, \ldots, \mathbf{g}^{q}$ and $\mathbf{a}^{q}$ are updated by ( $8 \mathrm{~b})$ and (10), respectively, for each $p=1, \ldots, N$, and then $\Phi^{q}(\widehat{\mathbf{r}})$ is updated by (12). Since, at each step, both (10) and (12) minimize $d^{2}(\mathbf{a}, \boldsymbol{\Phi})$, it results in $d^{2}\left[\mathbf{a}^{0}, \Phi^{0}(\widehat{\mathbf{r}})\right] \geq$ $d^{2}\left[\mathbf{a}^{1}, \Phi^{1}(\widehat{\mathbf{r}})\right] \geq \cdots \geq d^{2}\left[\mathbf{a}^{q}, \Phi^{q}(\widehat{\mathbf{r}})\right] \geq \cdots$. Therefore, the sequence $\left\{d_{q}^{2}\right\}=\left\{d^{2}\left[\mathbf{a}^{q}, \Phi^{q}(\widehat{\mathbf{r}})\right]\right\}$ is nonincreasing and thus convergent. This sequence is terminated when $\left(d_{q}^{2}-\right.$ $\left.d_{q+1}^{2}\right) / d_{q+1}^{2} \leq \varepsilon$, where $\varepsilon$ is a suitable threshold. Note that the proposed method has the considerable advantage of solving a problem characterized by multiple requirements (pattern shaping, polarization selection, and DRR reduction) with a low computational cost, thanks to the availability of the closed forms (10) and (12). However, it is a local search algorithm; thus the final solution may be dependent on the starting point $\mathbf{a}^{0}$. This latter aspect will be discussed in Section 4 by numerically analyzing the impact of the initial conditions on the final solution.

3.1. Phase-Only Control. The above presented algorithm allows one to control the DRR of the array excitations by properly selecting the nonnegative weight $b$ in (4). However, in order to simplify the feeding network, some applications require that the DRR be exactly equal to one, so that the joint pattern and polarization synthesis is realized by phaseonly control. To this aim, this subsection proposes a modified version of the algorithm in which all the excitations have the same amplitude. 
The problem can be reformulated by considering (7) with $b=0$ and $a_{n}=\zeta e^{j \psi_{n}}$ for $n=1, \ldots, N$, where $\zeta=\left|a_{1}\right|=\cdots=$ $\left|a_{N}\right|$. Thus, the objective becomes that of finding the common excitation amplitude $\zeta$, the column vector of the $N$ excitation phases $\psi=\left[\psi_{1}, \ldots, \psi_{N}\right]^{T}$, and the function $\Phi(\widehat{\mathbf{r}})$, in such a way as to minimize the functional

$$
d^{\prime 2}[\zeta, \psi, \Phi(\widehat{\mathbf{r}})]=\zeta^{2} \Psi^{H} \mathbf{A} \Psi-2 \zeta \Re\left(\mathbf{g}^{H} \boldsymbol{\Psi}\right)+\Upsilon^{2}
$$

where $\boldsymbol{\Psi}=\left[e^{j \psi_{1}}, \ldots, e^{j \psi_{N}}\right]^{T}$. An iterative approach can still be adopted to minimize (13), but now subdividing each iteration into three parts. In the first part, given $\psi$ and $\Phi(\widehat{\mathbf{r}}), d^{\prime 2}$ is minimized with respect to $\zeta$. To this aim, one can observe that $d^{\prime 2}$ is a parabolic function of $\zeta$; thus, $d^{\prime 2}$ is minimum for

$$
\zeta=\frac{\Re\left(\mathbf{g}^{H} \Psi\right)}{\Psi^{H} \mathbf{A} \Psi} .
$$

To this purpose, it is important to observe that both quantities $\boldsymbol{\Psi}^{H} \mathbf{A} \boldsymbol{\Psi}$ and $\mathcal{R}\left(\mathbf{g}^{H} \boldsymbol{\Psi}\right)$ are always positive because $\mathbf{a}^{H} \mathbf{A a}$ and $\mathfrak{R}\left(\mathbf{g}^{H} \mathbf{a}\right)$ in $(7)$ are positive for each $\mathbf{a} \in \mathbb{C}^{N}$. In fact, $\mathbf{a}^{H} \mathbf{A a}$ is the sum of the first and the third integral in (5), which are positive; furthermore, $2 \Re\left(\mathbf{g}^{H} \mathbf{a}\right)$ is equal to the second integral in (5), which is positive when recalling that the optimal function $\Phi(\widehat{\mathbf{r}})$ coincides with the phase of $E_{\theta}(\mathbf{a} ; \widehat{\mathbf{r}})$. This ensures that (14) always yields a positive value that minimizes (13).

In the second part of each iteration, given $\zeta$ and $\Phi(\widehat{\mathbf{r}}), d^{\prime 2}$ is minimized with respect to $\psi$ by modifying the excitation phases one at a time. Precisely, (13) can be manipulated to put into evidence its dependence on the generic $p$ th phase $\psi_{p}$, obtaining

$$
d^{\prime 2}[\zeta, \psi, \Phi(\widehat{\mathbf{r}})]=\rho_{p}+2 \zeta\left|\nu_{p}\right| \cos \left[\psi_{p}-\arg \left(\nu_{p}\right)\right],
$$

where $\rho_{p}$ is a term independent of $\psi_{p}$ and $\nu_{p}=\mathbf{A}_{p} \Psi_{p}-g_{p}$, with $\Psi_{p}=\left[e^{j \psi_{1}}, \ldots, e^{j \psi_{p-1}}, 0, e^{j \psi_{p+1}}, \ldots, e^{j \psi_{N}}\right]^{T}$. Hence, the functional in (15) is minimum for

$$
\psi_{p}=\arg \left(\nu_{p}\right)+(2 \kappa+1) \pi,
$$

where $\kappa$ is an arbitrary integer.

In the third part of each iteration, given $\zeta$ and $\psi, d^{\prime 2}$ is minimized with respect to $\Phi(\widehat{\mathbf{r}})$. In particular, by substituting (8b) into (13) and identifying as $\eta^{\prime}$ a term independent of $\Phi(\widehat{\mathbf{r}}), d^{\prime 2}$ can be expressed as

$$
d^{\prime 2}[\zeta, \psi, \Phi(\widehat{\mathbf{r}})]=\eta^{\prime}-2 \zeta \Re\left[\int_{\Theta} \Psi^{T} \widetilde{\mathbf{f}}(\widehat{\mathbf{r}}) E(\widehat{\mathbf{r}}) e^{-j \Phi(\widehat{\mathbf{r}})} \mathrm{d} \theta \mathrm{d} \phi\right]
$$

which is minimum for

$$
\Phi(\widehat{\mathbf{r}})=\arg \left[\Psi^{T} \widetilde{\mathbf{f}}(\widehat{\mathbf{r}})\right]
$$

The modified algorithm develops as follows. Moving from the starting common amplitude $\zeta^{0}$ and the starting vector of the excitation phases $\psi^{0}$, the function $\Phi^{0}(\widehat{\mathbf{r}})$ is evaluated by (18). At each successive iteration $q=1,2, \ldots$, once $\mathbf{g}^{q}$ is

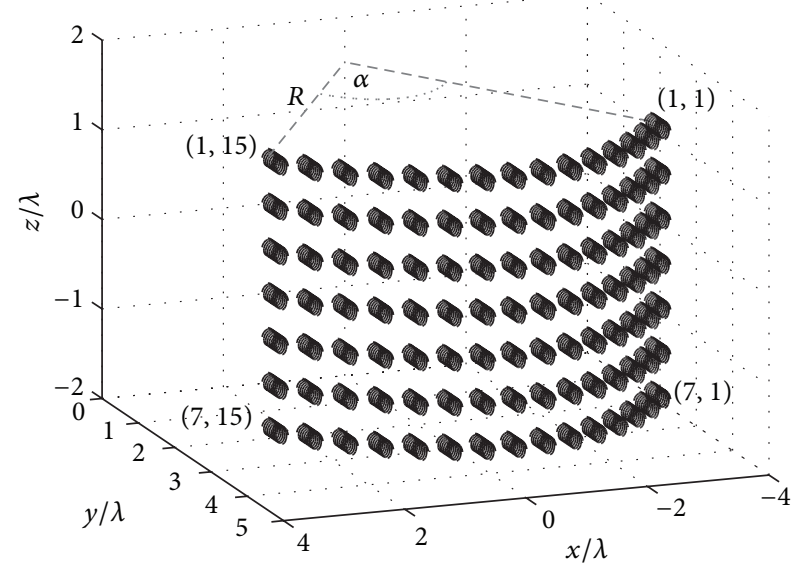

FIgURE 1: Cylindrical arc array of $N=15 \times 7$ quadrifilar helixes.

updated by (8b) for $p=1, \ldots, N$, the quantities $\zeta^{q}, \psi^{q}$, and $\Phi^{q}(\widehat{\mathbf{r}})$ are updated by (14), (16) for $p=1, \ldots, N$, and (18), respectively. The sequence $\left\{d_{q}^{\prime 2}\right\}=\left\{d^{\prime 2}\left[\zeta^{q}, \psi^{q}, \Phi^{q}(\widehat{\mathbf{r}})\right]\right\}$, which is still nonincreasing and hence convergent, is terminated when $\left(d_{q}^{\prime 2}-d_{q+1}^{\prime 2}\right) / d_{q+1}^{\prime 2} \leq \varepsilon^{\prime}$, where $\varepsilon^{\prime}$ is a suitable threshold.

\section{Numerical Results}

The performance of the proposed algorithm is evaluated by considering a conformal array consisting of $N=15 \times 7$ elements located on a cylindrical arc of radius $R=4.46 \lambda$ and aperture $\alpha=90^{\circ}$ (Figure 1). Each element is a quadrifilar helix antenna of radius $0.1 \lambda$ and turn spacing $0.2 \lambda$ with two turns and axis parallel to the $y$-axis. The generic helix lying in the $k$ th $\operatorname{arc}(k=1, \ldots, 7)$ and $h$ th column $(h=$ $1, \ldots, 15)$ of the array is placed at the point of cylindrical coordinates [ $R \sin [(22-h) \pi / 28], R \cos [(22-h) \pi / 28],(2-$ $k / 2) \lambda]$. This configuration is implemented in the SuperNEC 2.7 electromagnetic simulator to derive the single-element patterns $\widetilde{\mathscr{E}}_{n}(\widehat{\mathbf{r}})$ and $\widehat{\mathscr{E}}_{n}(\widehat{\mathbf{r}})$, in order to include the mutual coupling effects by adopting the approach described in [15].

The algorithm is implemented in MATLAB on an Intel Core2 Quad Q9300 @2.50 GHz Sun Ultra 24 workstation using just one core of the processor. A discretization step of 4 degrees in both the elevation and the azimuth domains is adopted; hence the desired directions and beamwidths are selected as multiples of 4 degrees. All results are derived by setting $\varepsilon=10^{-5}$ in absence of phase-only control, $\varepsilon^{\prime}=10^{-7}$ in presence of phase-only control, $w(\widehat{\mathbf{r}})=[E(\widehat{\mathbf{r}})]^{-\mu}$ with $\mu=6$ [11], and $v(\widehat{\mathbf{r}})=c \cdot 10^{\beta}$ with $\beta=7$ and $c$ taking different values.

As a first example, we consider a desired pattern $E(\widehat{\mathbf{r}})$ having a maximum side-lobe level SLL $=-10 \mathrm{~dB}$, and a maximum at $\left[\theta_{0}, \phi_{0}\right]=\left[88^{\circ}, 80^{\circ}\right]$, thus involving a desired direction close to the azimuth plane. The desired radiated far-field vector must be right-hand circularly polarized $(\delta=$ $1, \xi=-\pi / 2)$ within the main lobe $\Theta_{\mathrm{ML}}=\left[76^{\circ}, 100^{\circ}\right] \times$ $\left[72^{\circ}, 88^{\circ}\right]$, and the starting points are $\mathbf{a}^{0}=[0, \ldots, 0]^{T}$ in absence of phase-only control, and $\zeta^{0}=0, \psi^{0}=[0, \ldots, 0]^{T}$ in presence of phase-only control. Figure 2 presents the $2 \mathrm{D}$ 


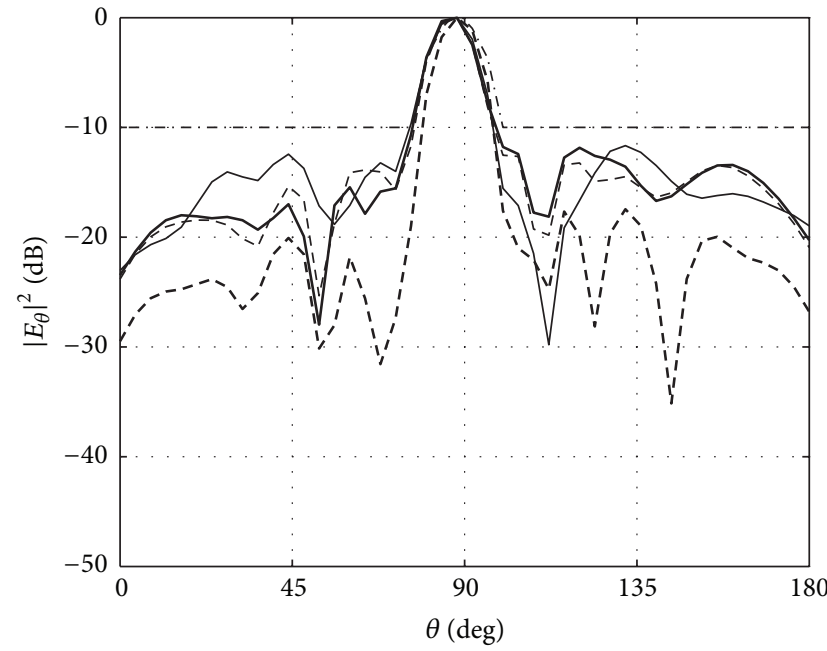

(a)

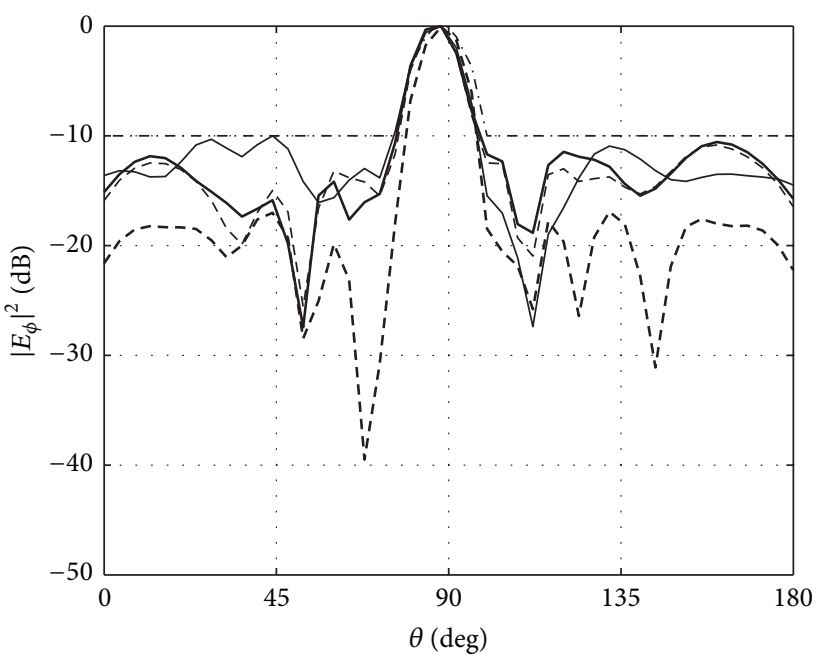

(c)
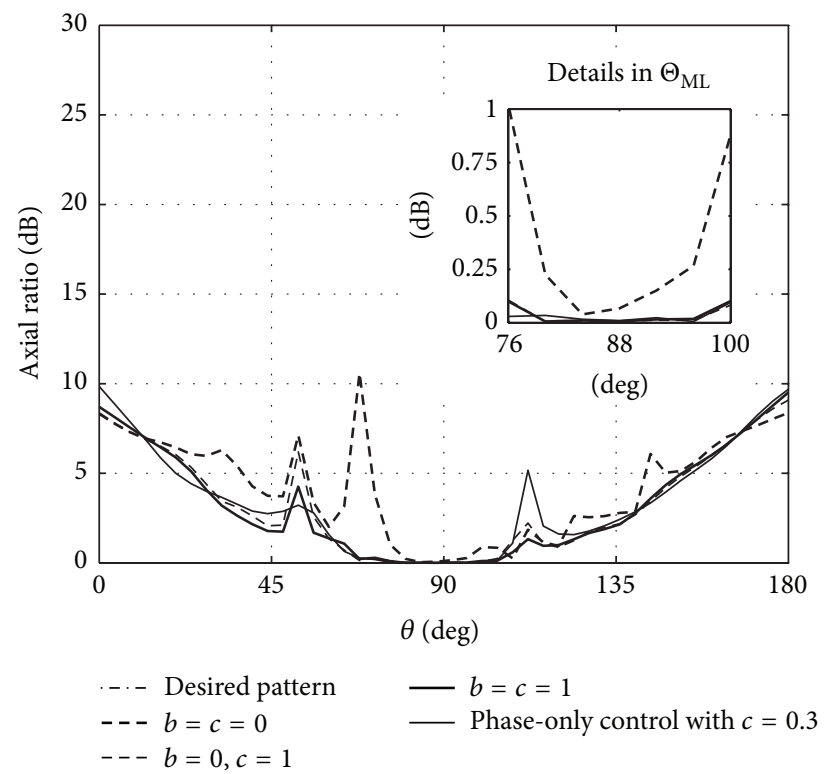

(e)

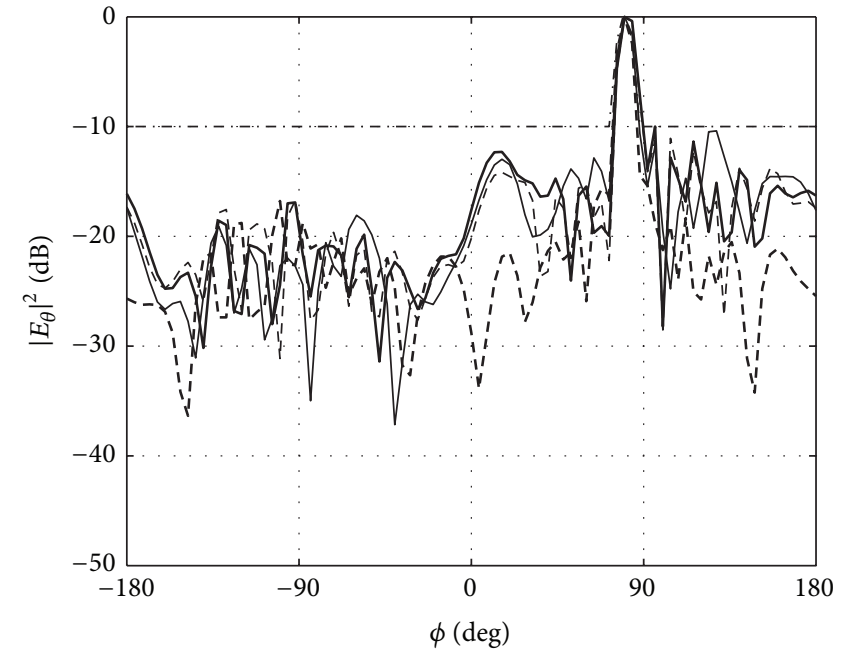

(b)

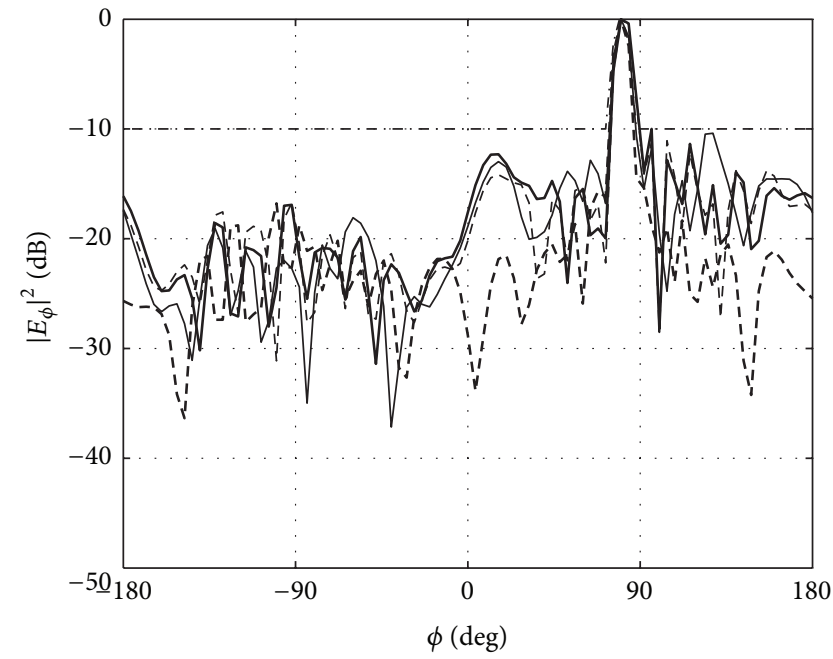

(d)

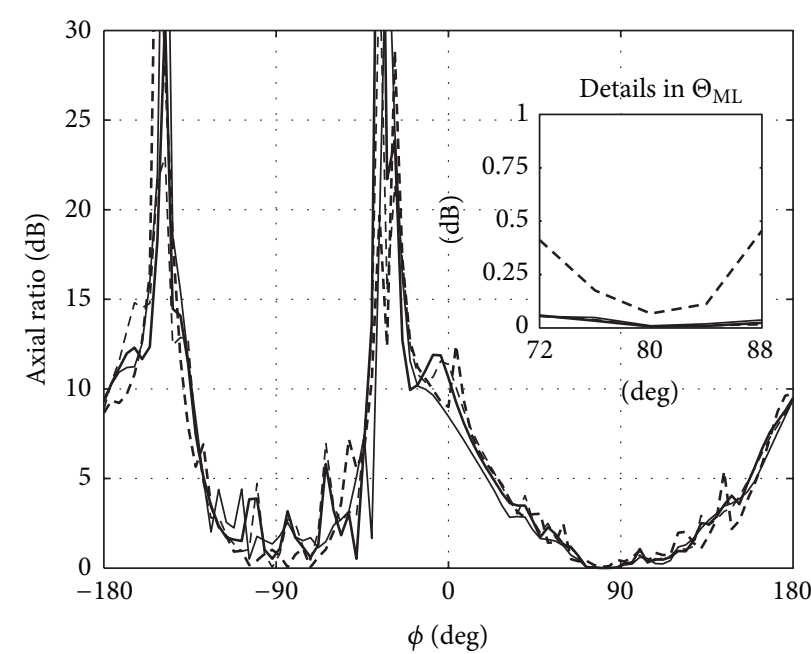

$\begin{array}{ll}-- \text { Desired pattern } & -b=c=1 \\ -- & b=c=0 \\ -- & b=0, c=1\end{array}$

(f)

Figure 2: Patterns and axial ratios obtained using the proposed algorithm: (a) $\left|E_{\theta}\right|^{2}$ for $\phi=\phi_{0}=80^{\circ}$, (b) $\left|E_{\theta}\right|^{2}$ for $\theta=\theta_{0}=88^{\circ}$, (c) $\left|E_{\phi}\right|^{2}$ for $\phi=\phi_{0}=80^{\circ}$, (d) $\left|E_{\phi}\right|^{2}$ for $\theta=\theta_{0}=88^{\circ}$, (e) axial ratio for $\phi=\phi_{0}=80^{\circ}$, and (f) axial ratio for $\theta=\theta_{0}=88^{\circ}$. 
TABLE 1: Performance of the proposed algorithm.

\begin{tabular}{lcccc}
\hline & $b=0$ & $b=0$ & $b=1$ & $\begin{array}{c}\text { Phase-only control } \\
\text { with } c=0.3\end{array}$ \\
& $c=0$ & $c=1$ & $c=1$ & -10.0 \\
\hline $\begin{array}{l}\text { SLL [dB] } \\
\text { Maximum axial ratio } \\
\text { in the main lobe [dB] }\end{array}$ & 2.7 & 0.1 & 0.1 & 0.2 \\
DRR & 99.8 & 60.8 & 7.9 & 1.0 \\
CPU time [s] & 4.6 & 9.3 & 6.1 & 312.1 \\
\hline
\end{tabular}

cuts, corresponding to $\theta_{0}$ and $\phi_{0}$, of the power patterns $\left|E_{\theta}\right|^{2}$ (Figures 2(a) and 2(b)) and $\left|E_{\phi}\right|^{2}$ (Figures 2(c) and 2(d)), and of the axial ratios with a magnification of the main lobe region (Figures 2(e) and 2(f)), while Table 1 reports the performance of the proposed algorithm for different values of the parameters. We remark that a $3 \mathrm{D}$ synthesis has been performed; thus the values in the table refer to the entire 3D domain, while the curves in Figure 2 refer to $2 \mathrm{D}$ cuts to maintain readability. The results show that in all the considered cases the pattern synthesized approximates the desired one. It is important to note that, even if the quadrifilar helix is an antenna designed to provide a circular polarization, when several helixes are used to form a conformal array, satisfying the requirements on the shape of the radiated far-field pattern might lead to a deterioration of the desired polarization state (case $b=c=0$ in Figures 2(e) and 2(f)). This effect can be considerably reduced using the proposed algorithm. In fact, in the presented application, the polarization within $\Theta_{\mathrm{ML}}$ can be controlled by properly selecting the parameter $c$. Precisely, when $c=1$, the axial ratio inside the main lobe is equal to $0.1 \mathrm{~dB}$ and hence a righthand circular polarization is substantially obtained, while, additionally, when $b=1$, a DRR reduction in the order of $87 \%$ is achieved. The results reveal also that the possibility of setting the values of the parameters $b$ and $c$ to match a specific requirement provides a considerable versatility to the proposed method, which furthermore requires a low CPU time, considering that just few seconds have been sufficient to perform the 3D synthesis of an array with $N=105$ elements. The last column of Table 1 shows that the modified algorithm is able to satisfy the requirement on the SLL maintaining an axial ratio of $0.2 \mathrm{~dB}$ when the synthesis must be performed by phase only control. In this case, the computational time increases to few minutes because a lower threshold $\varepsilon^{\prime}$ is required. This behavior is confirmed by Table 2 , which shows the impact of the starting point $\mathbf{a}^{0}$ on the performance of the presented algorithm for $b=c=1$ and of the starting point $\left(\zeta^{0}, \psi^{0}\right)$ on the performance of the modified algorithm for $c=0.3$. The values in the table, which are written in the form $\bar{x} \pm \sigma$, where $\bar{x}$ denotes the mean value and $\sigma$ the standard deviation, are obtained by performing one hundred realizations. The amplitudes $\zeta^{0}$ and $\zeta_{p}^{0}$, for $p=1, \ldots, N$, are generated according to a uniform distribution inside the interval $[0,1]$, while the phases $\psi_{p}^{0}$, for $p=1, \ldots, N$, are generated according to a uniform distribution inside the interval $[-\pi, \pi]$. The results in the table show, in particular for the case $b=c=1$, that the standard deviations referred
TABLE 2: Impact of the starting point on the results.

\begin{tabular}{|c|c|c|}
\hline & $\begin{array}{l}b=1 \\
c=1\end{array}$ & $\begin{array}{c}\text { Phase-only control } \\
\text { with } c=0.3\end{array}$ \\
\hline SLL $[\mathrm{dB}]$ & $-10.97 \pm 0.81$ & $-8.94 \pm 1.26$ \\
\hline $\begin{array}{l}\text { Maximum axial ratio } \\
\text { in the main lobe }[\mathrm{dB}]\end{array}$ & $0.17 \pm 0.07$ & $0.48 \pm 0.30$ \\
\hline DRR & $11.89 \pm 4.36$ & $1.00 \pm 0.00$ \\
\hline CPU time $[\mathrm{s}]$ & $6.99 \pm 1.86$ & $206.82 \pm 52.17$ \\
\hline
\end{tabular}

TABLE 3: Results obtained for the two-dimensional scanning of the region $\left[80^{\circ}, 100^{\circ}\right] \times\left[80^{\circ}, 100^{\circ}\right]$.

\begin{tabular}{lcc}
\hline & $b=c=0$ & $b=c=0.2$ \\
\hline SLL [dB] & $-15.00 \pm 0.70$ & $-10.75 \pm 2.59$ \\
Maximum axial ratio & $1.94 \pm 0.59$ & $0.42 \pm 0.25$ \\
in the main lobe [dB] & $65.56 \pm 49.27$ & $32.44 \pm 15.73$ \\
DRR & $4.68 \pm 1.24$ & $4.48 \pm 0.95$ \\
CPU time [s] & \multicolumn{1}{c}{ Phase-only control } \\
\hline \multicolumn{2}{c}{$c=0$} & $c=0.1$ \\
\hline SLL [dB] & $-14.03 \pm 1.12$ & $-9.89 \pm 2.44$ \\
Maximum axial ratio & $1.71 \pm 0.95$ & $0.80 \pm 0.29$ \\
in the main lobe [dB] & $1.00 \pm 0.00$ & $1.00 \pm 0.00$ \\
DRR & $131.50 \pm 24.01$ & $146.12 \pm 32.92$ \\
CPU time [s] &
\end{tabular}

to as the SLL and the axial ratio are sufficiently low, thus revealing that, for different starting points, even if different acceptable solutions are obtained, they substantially converge to a similar performance.

As a second example, we consider a two-dimensional scanning application, which requires the generation of a family of $6 \times 6=36$ patterns able to scan the angular region $\left[80^{\circ}, 100^{\circ}\right] \times\left[80^{\circ}, 100^{\circ}\right]$ by steps of 4 degrees, according to the adopted discretization. The generic desired pattern $E^{i, l}(\widehat{\mathbf{r}})$ of this family has a maximum at $\left[\theta_{0}^{i}, \phi_{0}^{l}\right]=\left[80^{\circ}+i \cdot 4^{\circ}, 80^{\circ}+l \cdot 4^{\circ}\right]$ $(i, l=0, \ldots, 5)$, and SLL $=-10 \mathrm{~dB}$. The desired radiated far-field vector must still be right-hand circularly polarized within the main lobe $\Theta_{\mathrm{ML}}^{i, l}=\left[\theta_{0}^{i}-12^{\circ}, \theta_{0}^{i}+12^{\circ}\right] \times\left[\phi_{0}^{l}-8^{\circ}, \phi_{0}^{l}+\right.$ $\left.8^{\circ}\right]$, and the starting points are $\mathbf{a}^{0}=[0, \ldots, 0]^{T}$ in absence of phase-only control and $\zeta^{0}=0, \psi^{0}=[0, \ldots, 0]^{T}$ in presence of phase-only control. Table 3 shows the obtained results, still referred to as the 3D domain and reported in the form $\bar{x} \pm \sigma$, where the mean value $\bar{x}$ and the standard deviation $\sigma$ are evaluated by averaging over the 36 patterns necessary to perform the scanning. The values in the table are derived considering four cases: $b=c=0$ and $b=c=0.2$ in absence of phase-only control; $c=0$ and $c=0.1$ in presence of phaseonly control. Besides, to provide a view of the synthesized patterns for the most challenging case, in which the scanning is performed by both polarization and phase-only control, Figure 3 presents the 2D cuts, corresponding to $\theta=88^{\circ}$ and $\phi=88^{\circ}$, of the power patterns $\left|E_{\theta}\right|^{2}$ (Figures 3(a) and 3(b)) and $\left|E_{\phi}\right|^{2}$ (Figures 3(c) and 3(d)). 


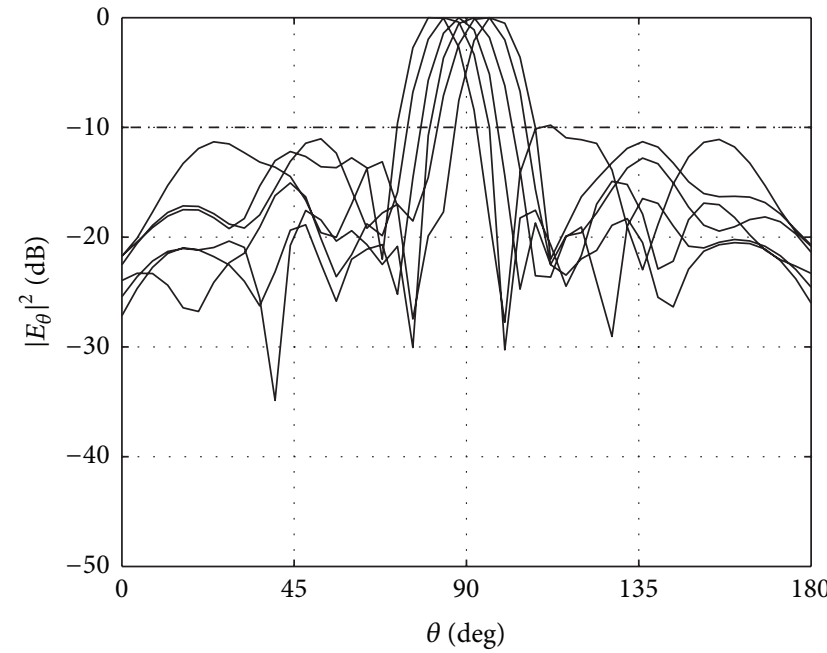

(a)

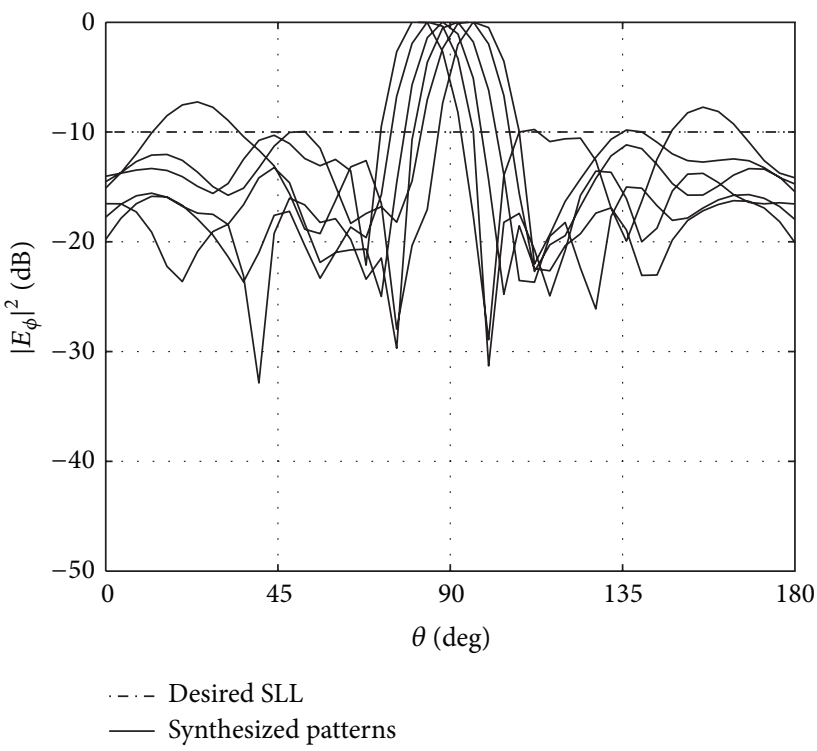

(c)

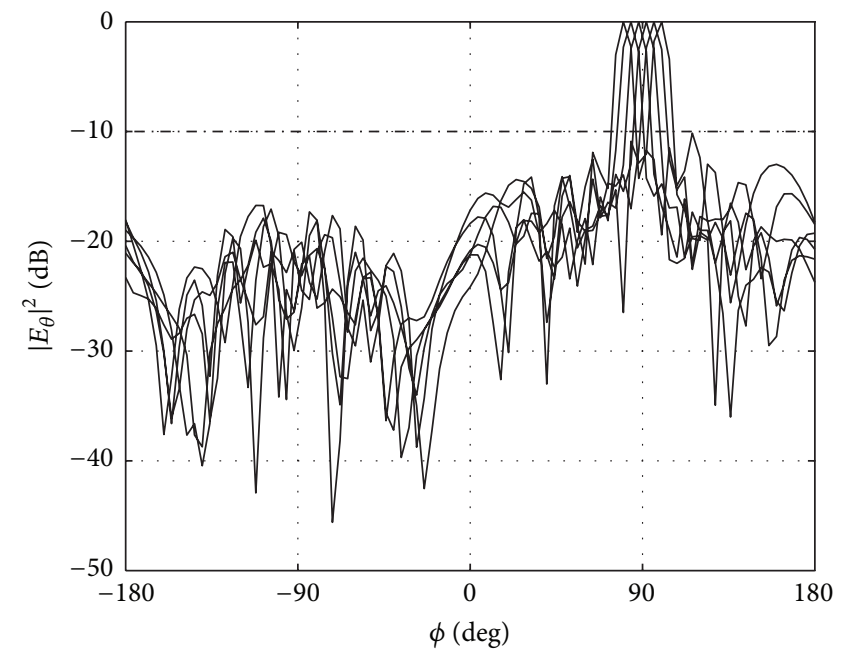

(b)

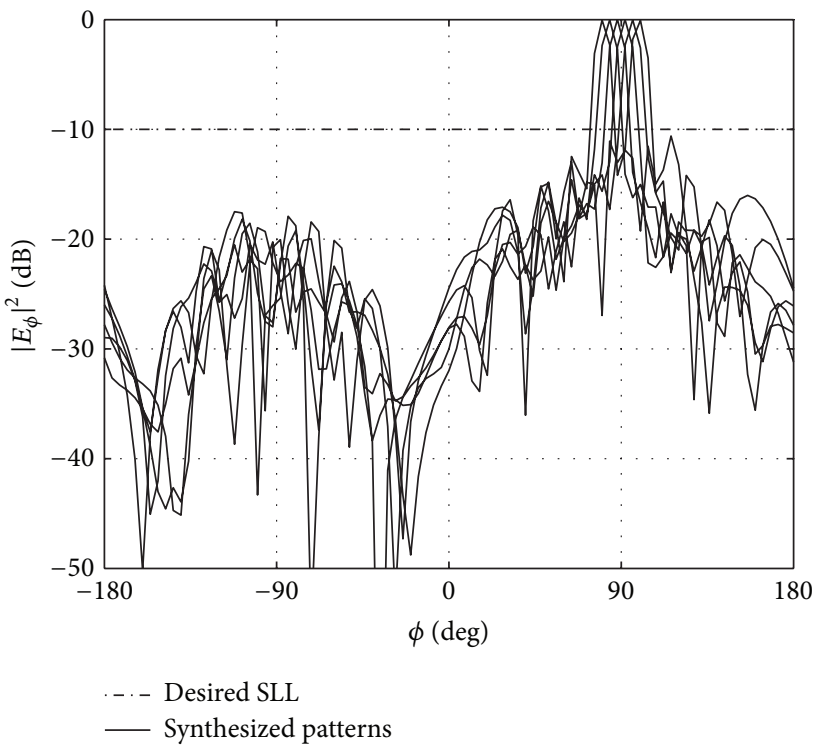

(d)

FiguRE 3: Patterns obtained for a two-dimensional scanning of the region $\left[80^{\circ}, 100^{\circ}\right] \times\left[8^{\circ}, 100^{\circ}\right]$ using the proposed algorithm by phase-only control with $c=0.1$ : (a) $\left|E_{\theta}\right|^{2}$ for $\phi=88^{\circ}$, (b) $\left|E_{\theta}\right|^{2}$ for $\theta=88^{\circ}$, (c) $\left|E_{\phi}\right|^{2}$ for $\phi=88^{\circ}$, and (d) $\left|E_{\phi}\right|^{2}$ for $\theta=88^{\circ}$.

These results reveal the satisfactory scanning capabilities of the proposed algorithm, which can be maintained also when improvements on the purity of the polarization and reductions of the DRR are required. In particular, one may observe from Figure 3 that the main lobe remains properly shaped even when a unitary DRR is imposed. In this regard, it is worth noticing that, during the scanning process, as the desired direction becomes farther from the direction identified by the $y$-axis, which is parallel to the main axis of each quadrifilar helix that composes the array, more difficulties arise in achieving a low axial ratio. In fact, in absence of polarization requirements, the scanned region can be considerably extended both in the elevation and the azimuth domain, obtaining satisfactory patterns both in absence and in presence of phase-only control. This possibility is confirmed by Table 4 , which presents the results derived maintaining, with respect to the previous case, the same starting points and pattern requirements in terms of beamwidth, shape of the main lobe, and SLL, but setting the parameter $c$ equal to zero and considering a two-dimensional scanning of the region $\left[64^{\circ}, 116^{\circ}\right] \times\left[64^{\circ}, 116^{\circ}\right]$ by steps of 4 degrees. The SLL values in the table and the $2 \mathrm{D}$ cuts of the power patterns $\left|E_{\theta}\right|^{2}$ and $\left|E_{\phi}\right|^{2}$, reported in Figures 4(a) and 4(b) and Figures 4(c) and 4(d), respectively, show that the main lobe remains properly shaped even with a phase-only control approach and a larger scanning region, which almost completely covers the azimuthal aperture of the cylindrical arc array. 


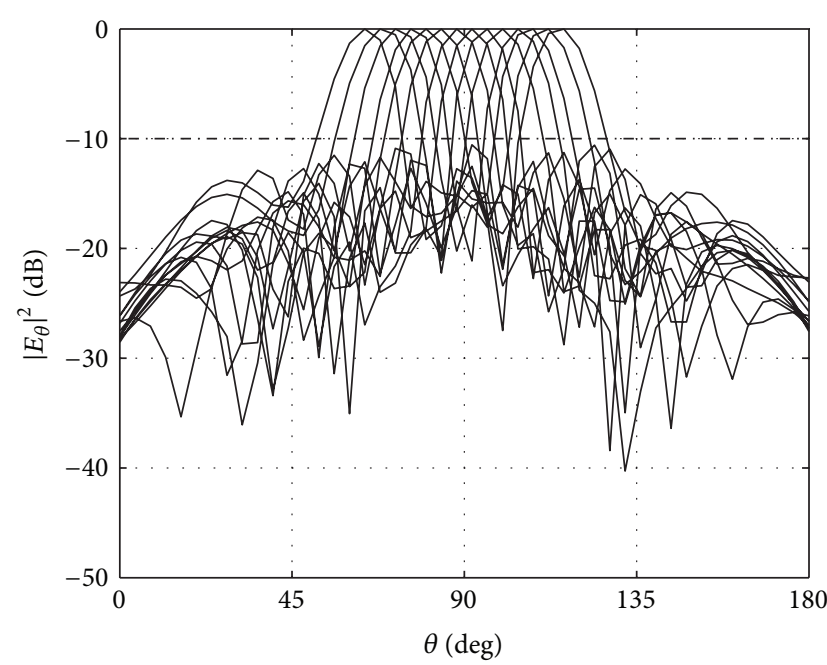

(a)

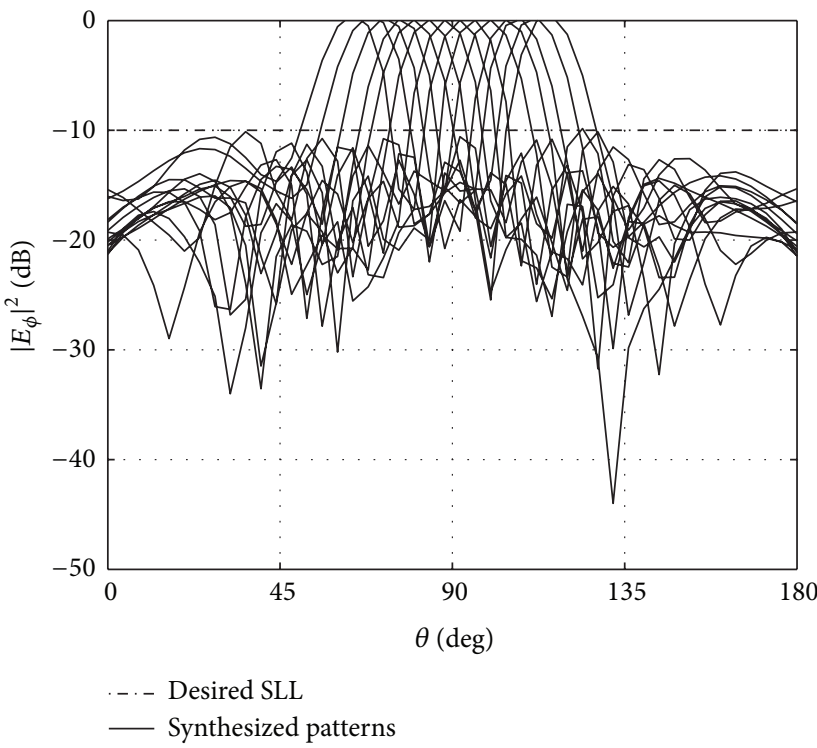

(c)

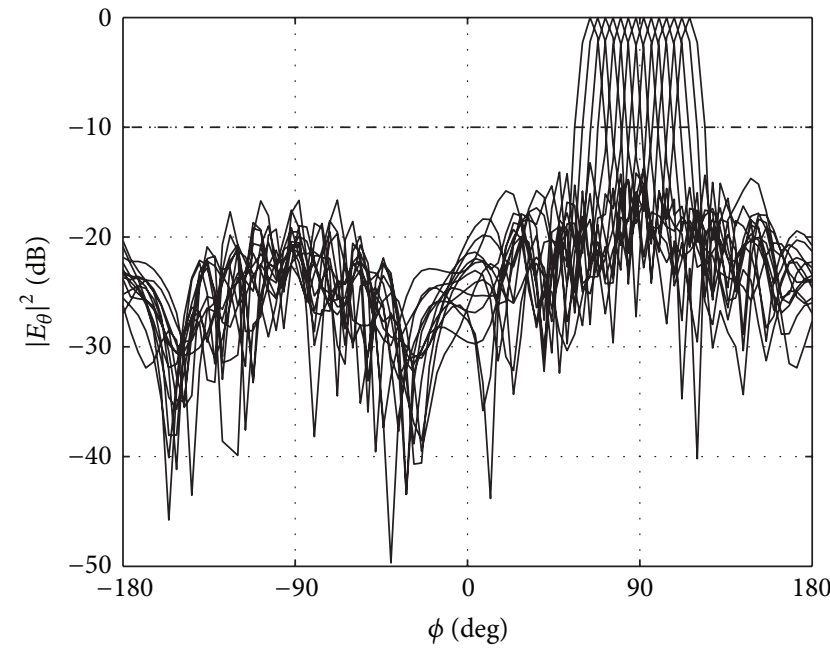

(b)

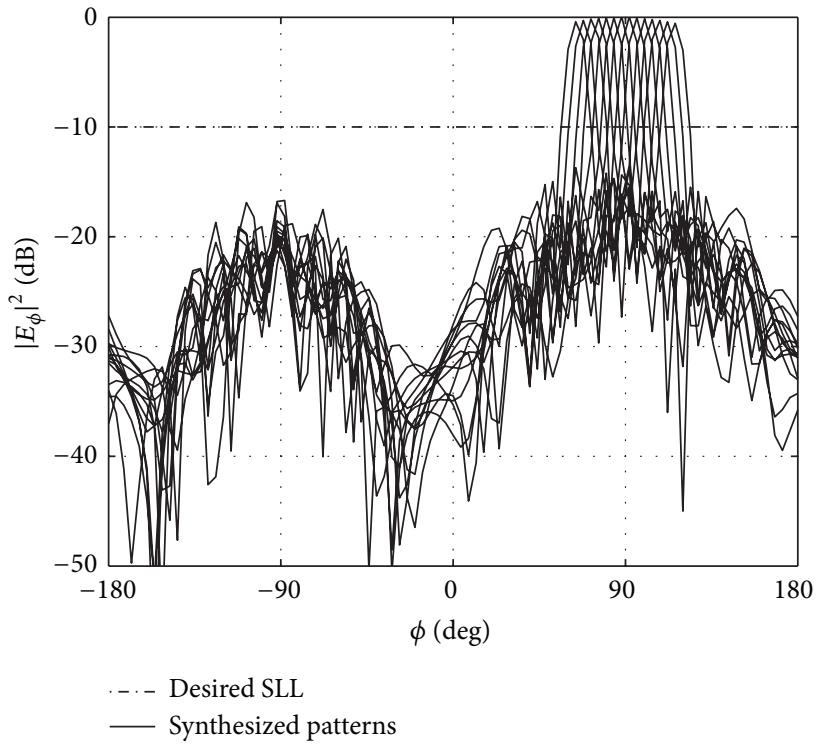

(d)

Figure 4: Patterns obtained for a two-dimensional scanning of the region $\left[64^{\circ}, 116^{\circ}\right] \times\left[64^{\circ}, 116^{\circ}\right]$ using the proposed algorithm by phase-only control with $c=0$ : (a) $\left|E_{\theta}\right|^{2}$ for $\phi=88^{\circ}$, (b) $\left|E_{\theta}\right|^{2}$ for $\theta=88^{\circ}$, (c) $\left|E_{\phi}\right|^{2}$ for $\phi=88^{\circ}$, and (d) $\left|E_{\phi}\right|^{2}$ for $\theta=88^{\circ}$.

TABLE 4: Results obtained for the two-dimensional scanning of the region $\left[64^{\circ}, 116^{\circ}\right] \times\left[64^{\circ}, 116^{\circ}\right]$.

\begin{tabular}{lcc}
\hline & $b=0.2$ & $\begin{array}{c}\text { Phase-only control } \\
\text { with } c=0\end{array}$ \\
\hline SLL [dB] & $-13.91 \pm 1.37$ & $-12.04 \pm 1.82$ \\
Maximum axial ratio & $2.72 \pm 1.39$ & $2.38 \pm 1.80$ \\
in the main lobe [dB] & $33.04 \pm 21.10$ & $1.00 \pm 0.00$ \\
DRR & $4.62 \pm 1.18$ & $140.19 \pm 34.06$ \\
CPU time [s] & & \\
\hline
\end{tabular}

\section{Conclusions}

A fast and versatile method for the joint pattern and polarization synthesis of conformal arrays when DRR or phase-only control is required has been presented. The method can be adapted to specific problems by properly selecting the available parameters, whose setting is not critical, as it requires just few attempts. The proposed algorithm can be applied both to arrays designed to support multiple polarizations, in order to select the desired one, and to arrays providing a unique polarization, in order to improve its purity by a proper choice of the excitations. Satisfactory results have also been obtained for two-dimensional scanning applications that involve the simultaneous control of the patterns' shape, of the polarization, and of the DRR.

\section{Conflict of Interests}

The authors declare that there is no conflict of interests regarding the publication of this paper. 


\section{Acknowledgments}

This work was supported in part by the Italian Ministry of University and Research (MIUR) within the Project FRA 2013 (University of Trieste, Italy), entitled "Multi-packet communication in $802.11 x$ heterogeneous mobile networks: models and antenna system algorithms."

\section{References}

[1] P. M. Haskins and J. S. Dahele, "Polarisation agile active microstrip patch arrays," Electronics Letters, vol. 32, no. 6, pp. 509-511, 1996.

[2] R. Vescovo, "Consistency of constraints on nulls and on dynamic range ratio in pattern synthesis for antenna arrays," IEEE Transactions on Antennas and Propagation, vol. 55, no. 10, pp. 2662-2670, 2007.

[3] S. Fu, Y. Cao, Y.Zhou, and S. Fang, "Improved low-profile helical antenna design for INMARSAT applications," International Journal of Antennas and Propagation, vol. 2012, Article ID 829371, 5 pages, 2012.

[4] K. Woelders and J. Granholm, "Cross-polarization and sidelobe suppression in dual linear polarization antenna arrays," IEEE Transactions on Antennas and Propagation, vol. 45, no. 12, pp. 1727-1740, 1997.

[5] V. Schejbal and V. Kovarik, "A method of cross-polarization reduction," IEEE Antennas and Propagation Magazine, vol. 48, no. 5, pp. 108-111, 2006.

[6] A. Vallecchi and G. B. Gentili, "Design of dual-polarized seriesfed microstrip arrays with low losses and high polarization purity," IEEE Transactions on Antennas and Propagation, vol. 53, no. 5, pp. 1791-1798, 2005.

[7] M. Thevenot, C. Menudier, A. El Sayed Ahmad et al., "Synthesis of antenna arrays and parasitic antenna arrays with mutual couplings," International Journal of Antennas and Propagation, vol. 2012, Article ID 309728, 22 pages, 2012.

[8] G. Oliveri, L. Lizzi, F. Robol, and A. Massa, "Polarizationagile ads-interleaved planar arrays," Progress in Electromagnetics Research, vol. 142, pp. 771-798, 2013.

[9] W. Bu-hong and G. Ying, "Frequency-invariant and low crosspolarization pattern synthesis for conformal array antenna," in IEEE Radar Conference (RADAR '08), pp. 1-6, 2008.

[10] R. K. Baee, K. Forooraghi, and S. Chamaani, "Conformal array pattern synthesis using a hybrid WARP/2LB-MOPSO algorithm," International Journal of Antennas and Propagation, vol. 2012, Article ID 202906, 7 pages, 2012.

[11] M. Comisso and R. Vescovo, "Fast co-polar and cross-polar $3 \mathrm{D}$ pattern synthesis with dynamic range ratio reduction for conformal antenna arrays," IEEE Transactions on Antennas and Propagation, vol. 61, no. 2, pp. 614-626, 2013.

[12] L. I. Vaskelainen, "Iterative least-squares synthesis methods for conformai array antennas with optimized polarization and frequency properties," IEEE Transactions on Antennas and Propagation, vol. 45, no. 7, pp. 1179-1185, 1997.

[13] B. Fuchs and J. J. Fuchs, "Optimal polarization synthesis of arbitrary arrays with focused power pattern," IEEE Transactions on Antennas and Propagation, vol. 59, no. 12, pp. 4512-4519, 2011.

[14] M. Comisso and R. Vescovo, "3D power synthesis with reduction of near-field and dynamic range ratio for conformal antenna arrays," IEEE Transactions on Antennas and Propagation, vol. 59, no. 4, pp. 1164-1174, 2011.
[15] R. Vescovo, "Null formation with excitation constraints in the pattern synthesis for circular arrays of antennas," Electromagnetics, vol. 21, no. 3, pp. 213-230, 2001. 

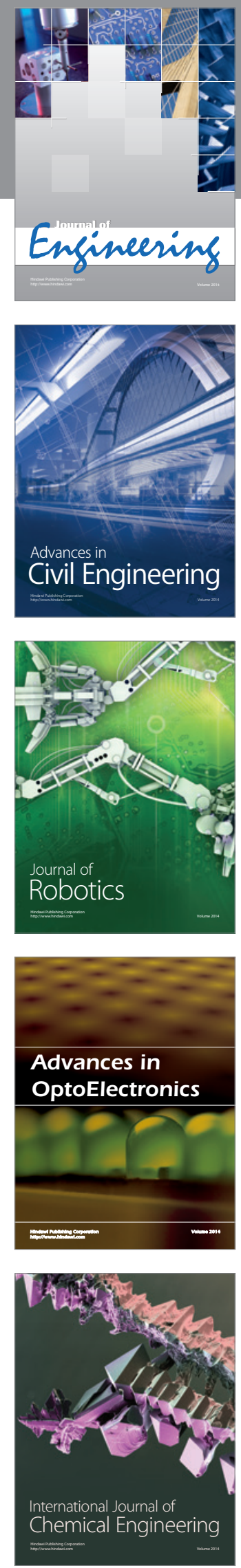

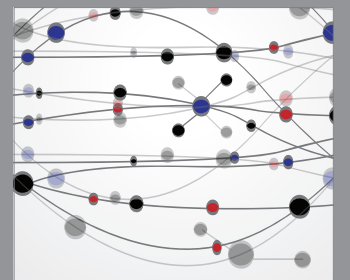

The Scientific World Journal
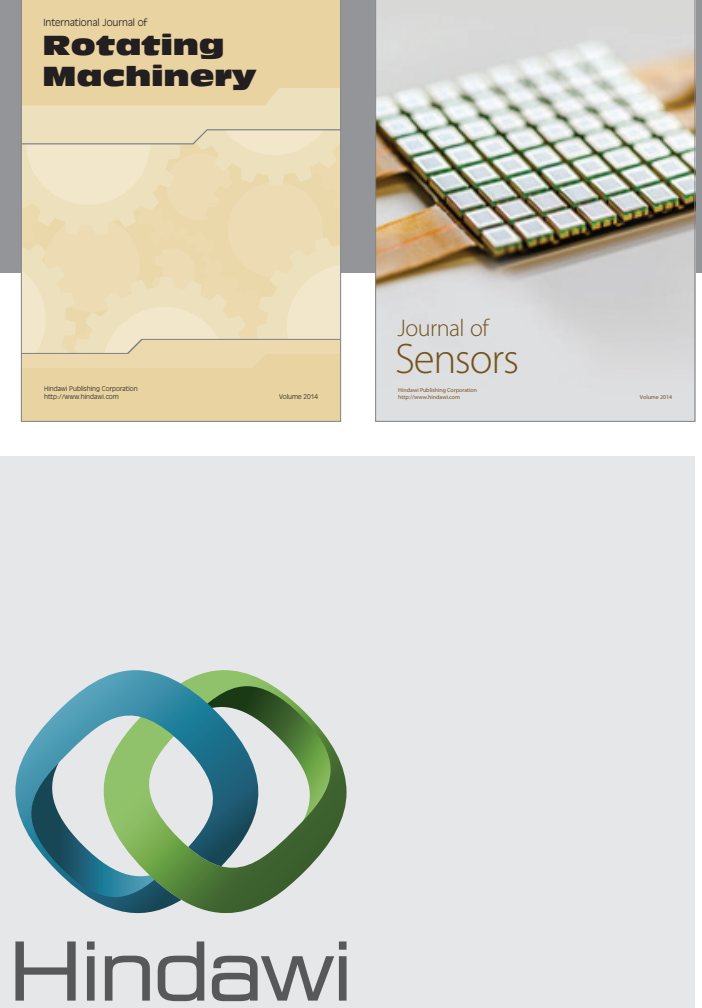

Submit your manuscripts at http://www.hindawi.com
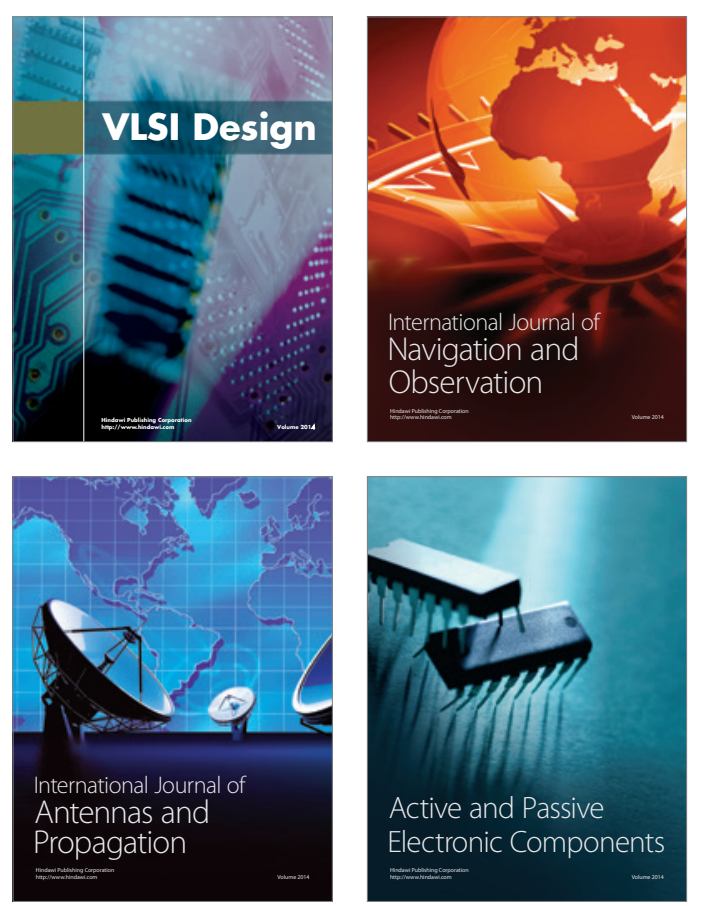
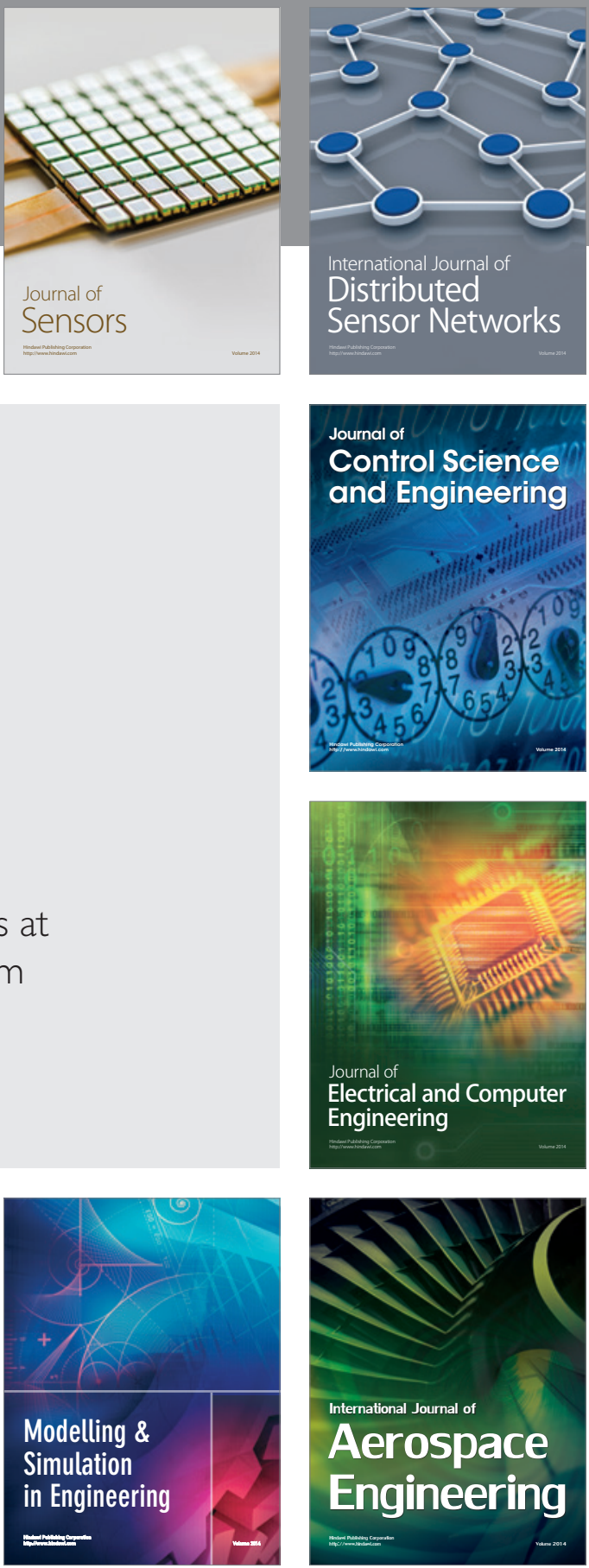

Journal of

Control Science

and Engineering
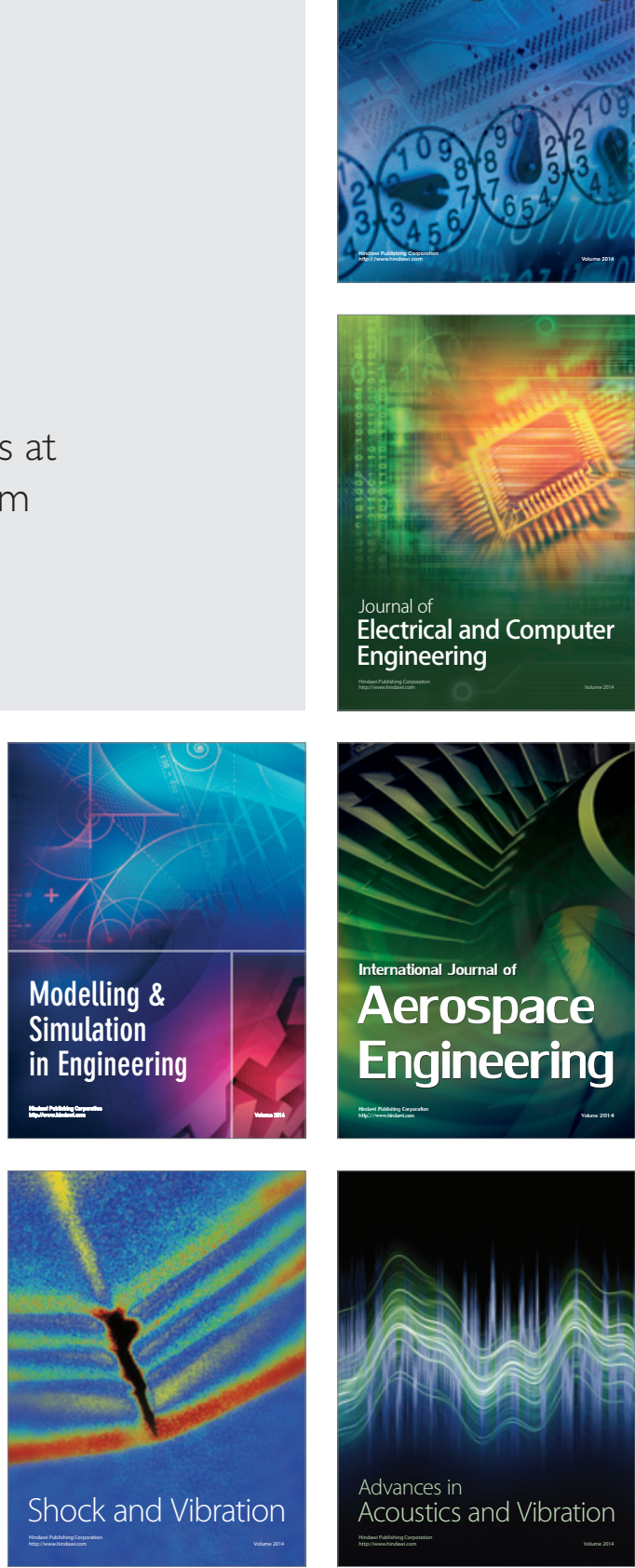